\title{
Mechanical Metastructure in Structural Engineering: A Short Review
}

\author{
Livija Cveticanin ${ }^{1,2 *}$ \\ ${ }^{1}$ University of Novi Sad, Serbia \\ ${ }^{2}$ Obuda University, Hungary
}

*Corresponding author: Livija Cveticanin, Faculty of Technical Sciences, University of Novi Sad, Serbia.

Received Date: July 29, 2020

Published Date: August 17, 2020

\begin{abstract}
In this paper a short review on the mechanical metastructure applied in civil and structural engineering is considered. This metastructure represents the macro version of the metamaterial which is a kind of composite. The main property of the mechanical metastructure is to mitigate and suppress vibration in systems. Depending on the configuration it represents vibration isolator or absorber which stops the low-frequency vibrations and forms an oscillation band-gap. Various types of mechanical metastructures with negative effective mass and negative stiffness are presented. In the paper the auxetic structures with negative Poison's coefficient for vibration elimination are also considered. The future investigation in the matter is suggested.
\end{abstract}

Keywords: Mechanical metastructure; Auxetic structure; Vibration isolator; Vibration absorber

\section{Introduction}

Vibration in structures represents an unwilling motion which negatively affect all living beings, primarily human ones, but also the entire working and living environment. There are two usual ways to combat pre-existing vibrations: reducing the amplitude of the oscillation (by installing an isolator) or eliminating the oscillation of a certain frequency (by a dynamic absorber). What both of these methods have in common is that they involve adding a mass or a mass-spring system to the existing structure. This causes variations in structure properties due to increase of the mass and the change of its rigidity. In addition, this intervention is not costless. Therefore, for the last 20 years, intensive work has been done on the development of new concepts of vibration reduction, but in a way to eliminate the mentioned shortcomings, without changing the functionality as well as the existing physical characteristics of the structure, above all, load-bearing capacity, strength and rigidity. Since 2000, research in this area has focused on finding materials that will be able to ensure the elimination of low-frequency oscillations in a certain range. It turned out that there are no such materials in nature, so they resorted to making artificial so-called metamaterials [1]. Meta-materials are composites whose structure is adjusted to meet certain requirements regarding physical properties. Thus, the idea came up to make a material of periodic structure consisting of a series of basic units, each of which contains a vibration absorber. Thus, instead of only one vibration absorber a large number of continually distributed absorbers have to be used to eliminate vibrations. This type of metamaterial is called mechanical or elastic. In the basic honeycomb structure absorbers, made of material which differs from the basic one, are incorporated. The fabrication of such a microscopic order structure proved to be technologically very difficult to do. However, the structure was realized at the macro level and an appropriate metastructure was formed. The metastructure is called mechanical or elastic. The 
paper [2] shows an aluminum chiral plate (dimensions $470 \times 91 \times 10$ $\mathrm{mm}$ ), in which rubber-coated metal cylinders are placed (Figure $1 \mathrm{~A})$.

Experimental measurements of the vibration characteristics of the structure with and without inserted absorbers were done. According to different layouts, different frequency responses are indicated, and the existence of a vibration gap i.e. absence of vibration for certain frequencies is evident. A theoretical explanation of this phenomenon is given in [3]. It was concluded that each of the basic units of the metastructure has a negative effective mass. The area of existence of the negative effective mass is directly related to the elimination of oscillations at a certain frequency. The larger the area of the negative effective mass, the larger the area for which this absorber ensures the absence of oscillations [4]. This area has been shown to be larger if the nonlinearity of the absorber is larger $[5,6]$.

\section{Discussion}

Three types of mechanical metastructures are considered: with negative effective mass, negative stiffness and negative Poison's ratio.

1. The appearance of elastic or mechanical metamaterials i.e. metastructures with added absorbers has attracted much attention from researchers $[7,8]$. The tests were aimed at determining the absorption power of the metastructure for different shapes and materials of the basic structure with a change in the position, shape and number of inserted absorbers (Figures 1B,1C). The disadvantage of these metastructures is the complexity of their fabrication. Hence, the requirement was set: material of the basic structure and absorbers has to be the same and that the metastructure has to be made as a single unit with negative effective mass [9].Thus, a structure made of polyurethane material was made on the 3D printer (Fig. 1g). The basic units of the structure are interconnected in the form of a 1D rod [10], a 2D plate [11] and a 3D form [12].

2. A metastructure that has a negative stiffness can also be used as a vibration isolator. Negative stiffness is manifested when the direction of force is not parallel to the direction of displacement in the basic structure [13]. The basic unit is a bistable structure that has two stable positions. The elastic deformation of the metastructure, plotted in Figure 2B, gives reversible energy of absorption. In [14], the metastructure of vibration isolators with basic units having quasi-zero dynamic stiffness is presented. The basic unit is formed as a sinusoidal rod and semicircular arches. This metastructure has significantly better vibration isolation capabilities than those exhibited by a linear isolator. The oscillation amplitude is significantly reduced with metastructure shown in Figure 3B. In addition, studies have shown that the vibration characteristic is better if the damping in the material is low and the excitation frequency is high (Figure 2,3).

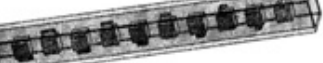

.

c)

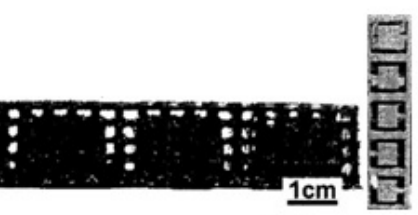

d)

Figure 1: Metastructure with vibroabsorbers: a) [2], b) [7], c) [8], d) [9]

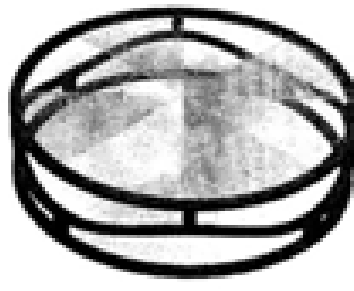

a)

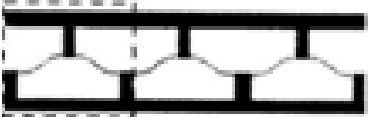

b)

Figure 2: a) Bistable base unit b) metastructure scheme [13]

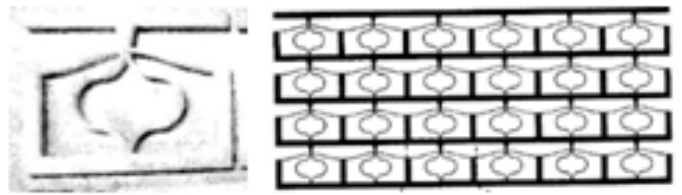

a)

b)

Figure 3: a) Base unit with zero stiffness b) metastructure scheme [14]. 
3. The structure that also shows good vibroabsorption properties is the one that has a negative Poisson's ratio. Such metastructures are called auxenic ones. The basic feature of these structures is that their dimension in the direction perpendicular to the direction of action of the tensile force increases and at the same time the structure absorbs energy. Basically, these structures are lattice with nodes (polygonal or circular) and elastic bending ligaments. Depending on the spatial arrangement of the nodes and ligaments, there are two basic unit structures [15]: one in which the ligaments are on the same or opposite side of the node. Fig.4a shows the metastructure with the anti-tetra base unit. In [16], a modification of the basic structure was performed in order to reduce the stress concentration in the nodes. Very often, a combination of tetra and anti-tetra units is used in the construction of the metastructure [17]. Depending on the number of ligaments, the basic structure can be four-sided or six-sided [18]. This metastructure is diluted according to the skin structure of lizards and snakes [19] (Figure 4). a)

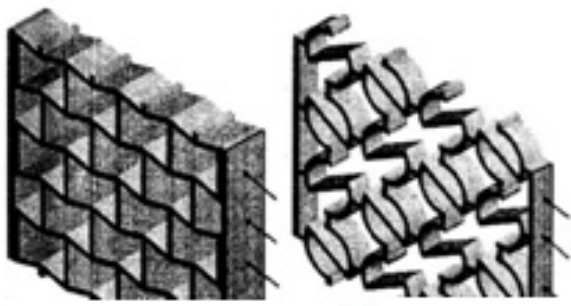

b)

Figure 4: a) Tetra lattice metastructure b) Modified S lattice metastructure [16].

\section{Conclusion}

Although the plate with metastructure has been shown to be very suitable as a vibration absorber or vibration isolator (depending on the structure), tests have also shown a major drawback. Namely, the structure shows anisotropy, i.e. when a force acts in one direction it shows an absorption property, but when a force acts in the other direction, this property does not exist. In addition, the characteristics of the slab with metastructure do not meet some of the requirements that designers usually set before it: they do not have sufficient strength, resistance to temperature changes, thermal conductivity, sufficient rigidity, etc. Hence, there is a need to harmonize the type of material and structure into a single whole in order to ensure: isotropy, suitability for load transfer, easy technical implementation i.e. production, resistance to temperature changes, but also low cost of fabrication.

\section{Acknowledgement}

None.

\section{Conflict of Interest}

No conflict of interest.

\section{References}

1. Cveticanin L, Mester G (2016) Theory of acoustic metamaterials and metamaterial beams: An Overview. Acta Polytechnica Hungarica 13(7): 43-62.

2. Zhu R, Liu XN, Hu GK, Sun CT, Huang GI (2014) A chiral elastic metamaterial beam for broadband. Journal of Sound and Vibration 333 2759-2773.

3. Milton GW (2007) New metamaterials with macroscopic behavior outside that of continuum elastodynamics. New Journal of Physics 9(359): 1-13.
4. Cveticanin L, Zukovic M, Cveticanin D (2018) On the elastic metamaterial with negative effective mass. Journal of Sound and Vibration 436: 295309.

5. Cveticanin L, Zukovic M (2014) Negative effective mass in acoustic metamaterial with nonlinear mass-in-mass subsystems. Communications in Nonlinear Science and Numerical Simulation 51: 89-104.

6. Cveticanin L, Zukovic M, Cveticanin D (2018) Influence of nonlinear subunits on the resonance frequency band gaps of acoustic metamaterial. Nonlinear Dynamics 93(3): 1341-1354.

7. Reichl KK, Inman DJ (2017) Lumped mass model of a 1D metastructure for vibration suppression with an additional mass. Journal of Sound and Vibration 403: 75-89.

8. Pierce CD, Willey CL, Chen VW, Hardin JO, Berrigan JD, et al. (2020) Adaptive elastic metastructures from magneto-active elastomers. Smart Material Structure 29(065004): 1-11

9. Hobeck JD, Laurent CMV, Inman DJ (2015) 3D prnting of metastructures for passive broadband vibration suppression. 20th Int Conf on Composite Materials Copenhagen Proc 1-8

10. Wang T, Sheng MP, Qin QH, (2016) Milti-flexural band gaps in an EulerBernoulli beam with lateral local resonators. Physics Letters A 380: 525529.

11. Peng H, Pai PF, Deng H (2016) Acoustic multi-stopband metamaterial plates design for broadband elastic wave absorption and vibration suppression. International Journal of Mechanical Sciences 109: 104-114.

12. Essink BC, Inman DJ. (2020) Three-dimensional mechanical metamaterial for vibration suppression. Special Topics in Structural Dynamics \& Experimental Techniques (Ed. N. Dervilia) 5: 43-48.

13. Zhang Y, Wang Q Tichem M, van Keulen F (2020) Design and characterization of multi-stable mechanical metastructures with level and tilted stable configurations. Extreme Mecahnics Letters 34(100593): 1-9.

14. Fan H, Yang L, Tian Y, Wang Z (2020) Design of metastructures with quasi-zero dynamic stiffness for vibration isolation. Composite Structures 243(112244) 1-13. 
15. Xia R, Song X, Sun L, Wu W, Li C, Cheng T, Qian G (2018) Mechanical properties of 3D isotropic anti-tetrachiral metastructure. Physica Status Solidi B 255(1700343): 1-9.

16. Meena K, Singamneni S (2019) A new auxetic structure with significantly reduced stress concentration effects. Materials and Design 173(107779): $1-11$.

17. Li H, Ma Y, Wen W, Wu W, Lei H, Fang D (2017) In plane mechanical properties of tetrachiral and antitetralchiral hybrid metastructures. Journal of Applied Mechanics 84 (081006): 1-11.
18. Mir M, Ali MN, Sami J, Ansaari U (2014) Review of Mechanics and applications of auxetic structures. Advances in Materials Science and Engineering Article ID 753496: 1-17.

19. Santulli C, Langella C (2016) Study and development of of concept of auxetic structures in bio-inspired design. International Journal of Sustainable Design 3(1): 20-37. 